\title{
Isoclinisms and covering groups
}

\section{Michael R. Jones and James Wiegold}

\begin{abstract}
The article examines the connection between the concepts of isoclinism and covering groups for finite groups. The main result is that all covering groups for a given finite group are mutually isoclinic. The converse is false.
\end{abstract}

\section{Introduction}

The idea of isoclinism was introduced by $\mathrm{Hall}$ in [2] as a means of classifying groups, and for completeness we reproduce the definition in 1.1 below. Covering groups for a given finite group were first defined by Schur in [5] and arose out of his work on projective representations of finite groups. For the reader who is unfamiliar with this notion, the definition may be found in 1.3 below. The aim of this note is to see how much connection there is between the ideas of covering group and isoclinism and it is found that another interesting problem concerning isoclinism arises. Throughout, the notation will be standard. In particular, the commutator $x^{-1} y^{-1} x y$ of two elements is denoted by $[x, y]$; the derived group and centre of a group $G$ are denoted by $G^{\prime}$ and $Z(G)$ respectively.

1.1. Let $G$ and $H$ be groups. Then $G$ and $H$ are said to be isoclinic if there exist isomorphisms $\xi$ and $\eta$ of $G / Z(G)$ onto $H / Z(H)$ and of $G^{\prime}$ onto $H^{\prime}$ respectively such that $[a, b] \eta=\left[a^{\prime}, b^{\prime}\right]$ whenever $(a Z(G)) \xi=a^{\prime} Z(H)$ and $(b Z(G)) \xi=b^{\prime} Z(H)$ for elements $a$ and $b$ of G. $\quad / /$

The following idea due to Neumann [4] will be of importance to us in our investigations:

Received 2 April 1974. 
1.2. Let $G$ and $H$ be groups and $\theta$ an epimorphism of $G$ onto $H$. Then $\theta$ is said to be an isoclinic epimorphism if $\operatorname{ker} \theta \cap G^{\prime}=1$. //

In the case of 1.2, $G$ and $H$ are found to be isoclinic (see [4]) a and $G$ is said to be an isoclinic ancestor of $H$.

1.3. Let $G$ be a finite group. A group $H$ is called a covering group of $G$ if there exists a subgroup $L$ of $H^{\prime} \cap Z(H)$ such that $H / L \cong G$ and $L \cong M(G)$, where $M(G)$ is the Schur multiplier of $G$ (see [3]).

It is well-known, and easy to see, that two covering groups of a given finite group need not be isomorphic. We shall see in the next section that they must at least be isoclinic. This fact unites and generalises the results of Schur [6], who established isomorphism of the commutator subgroups, and of Gaschütz, Neubüser und $\mathrm{Ti}$ Yen [1] giving isomorphism of the central factor-groups.

\section{Isoclinisms of covering groups}

THEOREM 2.1. AZZ the covering groups for a given finite group are mutually isoclinic.

Proof. Let $G$ be a finite group and let $F$ be a free group of finite rank such that $F / R \cong G$ for some normal subgroup $R$ of $F$. Let $H=F /[F, R], I=R /[F, R]$ and $T=\left(F^{\prime} \cap R\right) /[F, R]$.

Schur showed in [6] that, if $L$ is any covering group for $G$, there is an epimorphism $\phi$ from $H$ onto $L$ such that ker $\phi$ is a complement for $T$ in $I$. To prove the theorem, we need only observe that $\phi$ is an isoclinic epimorphism; and this is clear as

$$
\operatorname{ker} \phi \cap H^{\prime}=\operatorname{ker} \phi \cap H^{\prime} \cap L=\operatorname{ker} \phi \cap T=1 . \quad / /
$$

The existence of a common isoclinic ancestor as in the proof of Theorem 2.1 is not accidental, as the following result shows. It was originally proved in [7] using different methods.

PROPOSITION 2.2. Let $\left\{G_{i}: i \in I\right\}$ be any set of mutually isoclinic groups. Then there is a group $G$ which is an isoclinic ancestor for each $G_{i}$

Proof. We prove the result for $I=\{1,2\}$; the extension to any $I$ 
is obvious.

There exist isomorphisms $\xi$ of $G_{1} / Z\left(G_{1}\right)$ onto $G_{2} / Z\left(G_{2}\right)$ and $n$ of $G_{1}^{\prime}$ onto $G_{2}^{\prime}$ such that $[a, b] n=\left[a^{\prime}, b^{\prime}\right]$ whenever $\left(a Z\left(G_{1}\right)\right) \xi=a^{\prime} 2\left(G_{2}\right)$ and $\left(b 2\left(G_{1}\right)\right) \xi=b^{\prime} z\left(G_{2}\right)$.

Let $G$ be the subgroup of $G_{1} \times G_{2}$ consisting of those functions $f$ of $I$ into $G_{1} \cup G_{2}$ such that

$$
f(1) \phi_{1} \xi=f(2) \phi_{2}
$$

where $\phi_{j}$ is the natural epimorphism of $G_{j}$ onto $G_{j} / Z\left(G_{j}\right)$. Finally, let $\pi$ be the projection onto $G_{1}$ and $\alpha$ the restriction of $\pi$ to $G$. Then $\alpha$ is a homomorphism of $G$ into $G_{1}$. We show in two steps that $\alpha$ is an isoclinic epimorphism.

I. $\alpha$ is an epimorphism: Let $x$ be an element of $G_{1}$. Then there exists $y$ in $G_{2}$ such that

$$
y \phi_{2}=x \phi_{1} \xi
$$

Define $f$ on $I$ by the rule

$$
f(1)=x, f(2)=y \text {. }
$$

Then it is clear that $f$ belongs to $G$.

II. $\operatorname{ker} \alpha \cap G^{\prime}=1$ : Now, $G^{\prime}$ is generated by all $[f, g]$ where

$$
f(1) \phi_{1} \xi=f(2) \phi_{2} \text { and } g(1) \phi_{1} \xi=g(2) \phi_{2} \text {. }
$$

Then since $G_{1}$ and $G_{2}$ are isoclinic,

$$
[f(1), g(1)] \eta=[f(2), g(2)]
$$

so that, since $\eta$ is a homomorphism, $G^{\prime}$ consists of elements $h$ such that

$$
h(1) \in G_{1}^{\prime} \text { and } h(1) \eta=h(2) \text {. }
$$

If such an $h$ belongs to ker $\alpha, h(1)=1$ so that $h(2)=1$.

Hence $G$ is an isoclinic ancestor of $G_{1}$. Similarly, by considering 
$\xi^{-1}$ and $n^{-1}$, we may show that $G$ is an isoclinic ancestor of $G_{2} \cdot / /$

There is no converse to Theorem 2.1. That is, given a finite group and a covering group $B$ for $G$, it is not always true that every group isoclinic to $H$ is also a covering group for $G$, even if it is of the same order as $H$ (an obvious restriction). For suppose that $n$ is a natural number with $n \geq 3$, suppose $H$ is generalised quaternion of order $2^{n}$ and suppose $K$ is dihedral of order $2^{n}$. Then since $H$ has trivial multiplier (see [3]), it is a covering group for itself. But $K$ is isoclinic to $H$ and is clearly not a covering group for $H$.

However, we may pose the following problem:

PROBLEM 2.3. What finite groups $G$ are there such that if $H$ is a covering group for $G$ and $K$ is a group isoclinic to $H$ and of the same order as $H, K$ is also a covering group for $G$ ? //

The class of groups satisfying the conditions of Problem 2.3 is nonempty, as we shall now see.

LEMMA 2.4. Let $G$ be a finite perfect group and $H$ any finite group isoclinic with $G$. Then $H$ is isomorphic with the direct product of $G$ and $Z(H)$ amalgamating $H^{\prime} \cap Z(H)$ and $Z(G)$.

Proof. This is routine. //

COROLLARY 2.5 (Hall [2]). Let $G$ be a perfect group with trivial centre. Then any group isoclinic to $G$ is isomorphic with the direct product of $G$ and an abelian group. I/

PROPOSITION 2.6. Let $G$ be a finite perfect group and $H$ a group isoclinic to $G$ and of the same order as $G$. Then $H$ is isomorphic with $G$.

Proof. By Lemma 2.4, $H=\left(G_{1} \times A\right) / N$, where $|N|=|Z(G)|$ and $G_{1} \cong G$. Since $|H|=\left|G_{1}\right|, H=G_{1} \cong G$. $/ /$

An immediate corollary of Proposition 2.6 is the following, which goes some way towards solving Problem 2.3.

THEOREM 2.7. Let $G$ be a finite perfect group. Let $H$ be a covering group for $G$, let $K$ have the same order as $H$ and suppose $K$ 
is isoclinic with $H$. Then $K$ is isomorphic with $H$.

Proof. Since $G$ is perfect, it is easy to see that $H$ must also be perfect. Hence, by Proposition 2.6, $K \cong H$ and the result follows. //

Note here that any group $H$ as in Theorem 2.7 has trivial multiplier, whatever perfect $G$ we start with. This simple fact appears not to be recorded anywhere.

In view of Proposition 2.6 we may pose the following problem:

PROBLEM 2.8. For what groups $G$ does $|H|=|G|$ and $H$ isoclinic to $G$ imply $H \cong G$ ? $/ /$

Clearly, groups of the form $A \times B \times C$, where $A$ is perfect, $B$ is centreless and $C$ is cyclic of square-free order, have the property required by Problem 2.8. However, this is not the only class of groups with this property. For, suppose $G$ is the covering group of $A_{4}$, the alternating group on four letters. Then $\left|G^{\prime}\right|=8$ so that a sylow 2subgroup is normal. Now suppose $|K|=24$ and $K$ is isoclinic to $G$. Then $K^{\prime} \cong G^{\prime}$ so that $K^{\prime}$ is isomorphic with the quaternion group and an element of order 3 acts non-trivially on $K^{\prime}$. Hence $K \cong G$ so that $G$ , has the desired property.

\section{References}

[1] W. Gaschütz, J. Neubüser und Ti Yen, "über den Multiplikator von p-Gruppen", Math. Z. 100 (1967), 93-96.

[2] P. Hall, "The classification of prime-power groups", J. reine angew. Math. 182 (1940), 130-141.

[3] B. Huppert, Endliche Gruppen I (Die Grundlehren der mathematischen Wissenschaften, Band 134. Springer-Verlag, Berlin, Heidelberg, New York, 1967).

[4] B.H. Neumann, "Groups with finite classes of conjugate subgroups", Math. 2. 63 (1955), 76-96.

[5] J. Schur, "Über die Darstellungen der endlichen Gruppen durch gebrochene lineare Substitutionen", J. reine angew. Math. 127 $(1904), 20-50$. 
[6] J. Schur, "Untersuchungen über die Darstellungen der endlichen Gruppen durch gebrochene lineare Substitutionen", J. reine congew. Math. 132 (1907), 85-137.

[7] James Wiegold, "The derived groups of groups with boundedly finite conjugacy classes", (MSc thesis, University of Manchester, 1955).

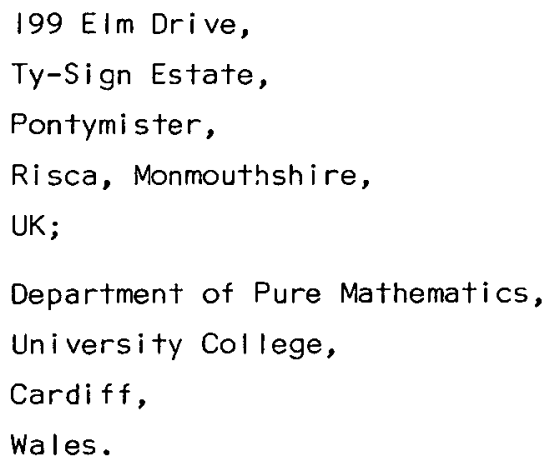

